\title{
Caracterización del melanoma maligno en la Clínica de Tumores de Piel y Mucosas, Universidad de Caldas, 2005-2015
}

Characterization of malignant melanoma from the Clínica de Tumores de Piel y Mucosas, Universidad de Caldas, 2005-2015

\section{Heliana Marcela Botello-Mojica¹, Ana Patricia Insuasty-Moreno², Felipe Jaramillo- Ayerbe $^{3}$}

1. Estudiante de profundización, XIII Semestre de Medicina, Universidad de Caldas, Manizales, Colombia

2. Médica, residente de Dermatología de tercer año, Universidad de Caldas, Manizales, Colombia

3. Médico dermatólogo; profesor titular de Dermatología y Dermatopatología; coordinador, posgrado de Dermatología, Universidad de Caldas, Manizales, Colombia

\section{RESUMEN}

Introducción. El melanoma maligno exhibe un comportamiento biológico agresivo, representa cerca del $4 \%$ de las neoplasias cutáneas malignas, causa el 80 $\%$ de la mortalidad por cáncer de piel y su incidencia aumenta exponencialmente en el tiempo.

Objetivo. Caracterizar el perfil epidemiológico, clínico e histológico de los pacientes con diagnóstico de melanoma maligno evaluados en la Clínica de Tumores de la Universidad de Caldas.

Materiales y métodos. Se llevó a cabo un estudio retrospectivo y descriptivo. Se revisaron las historias clínicas de 492 pacientes que asistieron a la Clínica de Tumores, entre mayo de 2005 y octubre de 2015; se analizaron las siguientes variables: nombre, sexo, edad, tipo clínico, asociación con nevo melanocítico congénito, tiempo de evolución, índice de Breslow, nivel de Clark, localización, ulceración, metástasis, estadio y tratamiento.

Resultados. Se analizaron 102 casos de melanoma maligno en 98 pacientes, el sexo femenino representó el 58,1 \% y la sexta década fue la edad de presentación más común. El melanoma lentiginoso distal (acral) fue el tipo más frecuente $(24,5$ $\%$ ), seguido del melanoma extensivo superficial (23,5\%); en promedio, el tiempo de evolución fue de 29,6 meses. El 37,7 \% de los casos tenían un índice de Breslow entre o y 1 mm, y el 37,2 \%, un nivel IV de Clark. El 23,4 \% se encontraban en estadio IV; la cirugía convencional fue el tratamiento más empleado.

Conclusiones. Los estudios colombianos muestran resultados discrepantes de las características epidemiológicas encontradas en este trabajo, por lo tanto, se sugiere la realización de estudios similares con una muestra poblacional que incluya diferentes departamentos y permita establecer un perfil epidemiológico representativo del país.

PALABRAS CLAVE: melanoma, neoplasias cutáneas, epidemiología.
Correspondencia:

Heliana marcela botello mojica

Email:

heliana91@hotmail.com

Recibido: 11/01/2017

Aceptado: 24/05/2017

Conflictos de interés:

No se reportan conflictos de interés.

Financiación:

Ninguna. 


\section{SUMMARY}

Introduction: Malignant melanoma exhibits aggressive biological behavior, accounting for about $4 \%$ of skin malignancies and 80\% of skin cancer mortality; its incidence increases exponentially over time.

Objective: To characterize the epidemiological, clinical and histological profile of patients evaluated at the Clínica de Tumores of the Universidad de Caldas with diagnosis of malignant melanoma.

Materials and methods: A retrospective and descriptive study was carried out. We reviewed the medical records of 492 patients attending the Clínica de Tumores between May, 2005, and October, 2015, and analyzed the following variables: name, gender, age, clinical type, association with congenital melanocytic nevus, time of evolution, Breslow index, Clark's level, localization, ulceration, metastasis, stage and treatment.

Results: We analyzed 102 cases of malignant melanoma in 98 patients, the female sex represented $58.1 \%$, and the sixth decade was the most common age of presentation. Acral lentiginous melanoma was the most frequent type (24.5\%), followed by superficial extensive melanoma (23.5\%).The average evolution time was 29.6 months. Thirty seven point seven per cent of the cases had Breslow indexes between o and $1 \mathrm{~mm}$, and $37.2 \%$ Clark's level IV. Twenty three point four per cent were found in stage IV. Conventional surgery was the treatment most commonly used.

Conclusions: In Colombia, previous studies show discrepant results with the epidemiological characteristics found in this study. So, we suggest to plan similar studies with a population sample that includes different states and allows establishing a representative epidemiological profile for the country.

KEY WORDS: Melanoma, skin neoplasms, epidemiology.

\section{"En Colombia, el Instituto Nacional de Cancerología ha reportado una incidencia anual de melanoma maligno de 731 y 590 casos en hombres y de 892 y 613 casos nuevos en mujeres, en los periodos de 2002 a 2006 y de 2007 a 2011, respectivamente; Bogotá es la ciudad con la tasa registrada más alta."}

\section{INTRODUCCIÓN}

El melanoma maligno es la proliferación maligna de melanocitos, un tumor que se caracteriza por exhibir un comportamiento biológico agresivo. Se puede presentar en cualquier tejido debido a la migración de melanocitos derivados de la cresta neural; en la piel, suele originarse a partir de los melanocitos ubicados en la capa basal de la epidermis, extendiéndose a las capas más superficiales y a la dermis subyacente, desde donde puede generar metástasis por vía linfática o hematógena.

Representa cerca del $4 \%$ de todos los tipos de cáncer de piel y alcanza hasta el $80 \%$ de las muertes por esta causa $^{(1)}$. Ha llegado a considerarse una epidemia en la población caucásica, dado que la incidencia a lo largo de 10 años en mujeres y hombres se ha incrementado en Australia de 12 a $22 \%$, en Estados Unidos de 25 a 31
$\%$ y en el Reino Unido de 41 a $59 \%{ }^{(2)}$. En Colombia, el Instituto Nacional de Cancerología ha reportado una incidencia anual de melanoma maligno de 731 y 590 casos en hombres y de 892 y 613 casos nuevos en mujeres, en los periodos de 2002 a 2006 y de 2007 a 2011, respectivamente; Bogotá es la ciudad con la tasa registrada más alta ${ }^{(3,4)}$; asimismo, se describe un incremento de 2,7 a 13 casos por 10.000 habitantes entre 2003 y 2005, en el Centro Dermatológico Federico Lleras Acosta en Bogotá, Colombia ${ }^{(5)}$, con un aumento en el número de casos nuevos anuales en el país. En el departamento de Caldas, la incidencia reportada entre 1982 y 1995 en la consulta externa fue de 1,6 por 100.00o pacientes nuevos por año ${ }^{(6)}$.

Los principales factores de riesgo son: la historia personal de melanoma, el síndrome de nevo displásico, el melanoma familiar relacionado con mutaciones en el los genes CDKN2A y CDK4 en algunos casos, la ex- 
posición solar intermitente, el número de quemaduras solares en niños y adolescentes, y los más propensos fueron los fototipos I y II; asimismo, se describen el xeroderma pigmentario, los estrógenos, la progesterona y los estados de inmunosupresión ${ }^{(2,7,8)}$.

Existen cinco formas principales de melanoma: melanoma lentigo maligno, melanoma extensivo superficial, melanoma nodular, melanoma lentiginoso distal (acral) y el melanoma mucoso. También, se conocen variantes clínicas, como el melanoma amelanótico, el desmoplásico y el polipoide, entre otras ${ }^{(9)}$.

Los factores de pronóstico más importantes en la supervivencia a largo plazo son el crecimiento vertical, medido de forma cuantitativa por el índice de Breslow, y la presencia o ausencia de metástasis ganglionares $(2,8,10)$.

Otros factores de pronóstico histológico son el nivel de Clark, la presencia de ulceración, la tasa mitótica y la reacción inflamatoria. Entre los factores de pronóstico clínicos, se encuentran la localización anatómica, la edad y el sexo ${ }^{(7,8)}$.

El tratamiento de elección es el quirúrgico y depende de la localización, del tipo de melanoma y del estadio. El conocimiento de la epidemiología del melanoma maligno en Colombia permitirá determinar su comportamiento en el país y entender mejor la enfermedad. Con el fin de dar continuidad al primer estudio realizado en el departamento de Caldas por uno de los autores del presente artículo y colaboradores, durante los años de 1982 a 1995, el objetivo de este trabajo fue caracterizar el perfil epidemiológico de los pacientes con diagnóstico de melanoma maligno atendidos en la Clînica de Tumores de Piel y Mucosas, de la Universidad de Caldas.

\section{MATERIALES Y MÉTODOS}

El presente estudio es retrospectivo y descriptivo. Se analizaron las historias clínicas de todos los pacientes de todas las edades atendidos en la Clínica de Tumores de Piel y Mucosas, del posgrado de Dermatología de la Universidad de Caldas, en el periodo comprendido entre mayo de 2005 y octubre de 2015.

Se incluyeron las historias clínicas con diagnóstico de melanoma maligno y se excluyeron aquellas que no tuvieran la información suficiente para responder las variables. La categorización del estadio del melanoma maligno se hizo con base en la clasificación propuesta por el American Joint Committee on Cancer (AJCC), en su séptima versión publicada en $2009^{(11)}$.

Se analizaron las siguientes variables: nombre, sexo, edad, tipo clínico, asociación con nevo melanocítico congénito, tiempo de evolución, índice de Breslow, nivel de Clark, localización, ulceración, metástasis, estadio y modalidad terapéutica. El análisis estadístico se realizó con el (software SPSS ${ }^{\mathrm{TM}}$, Statistics 21.0. Las variables se describieron mediante medidas de tendencia central y frecuencias.

\section{RESULTADOS}

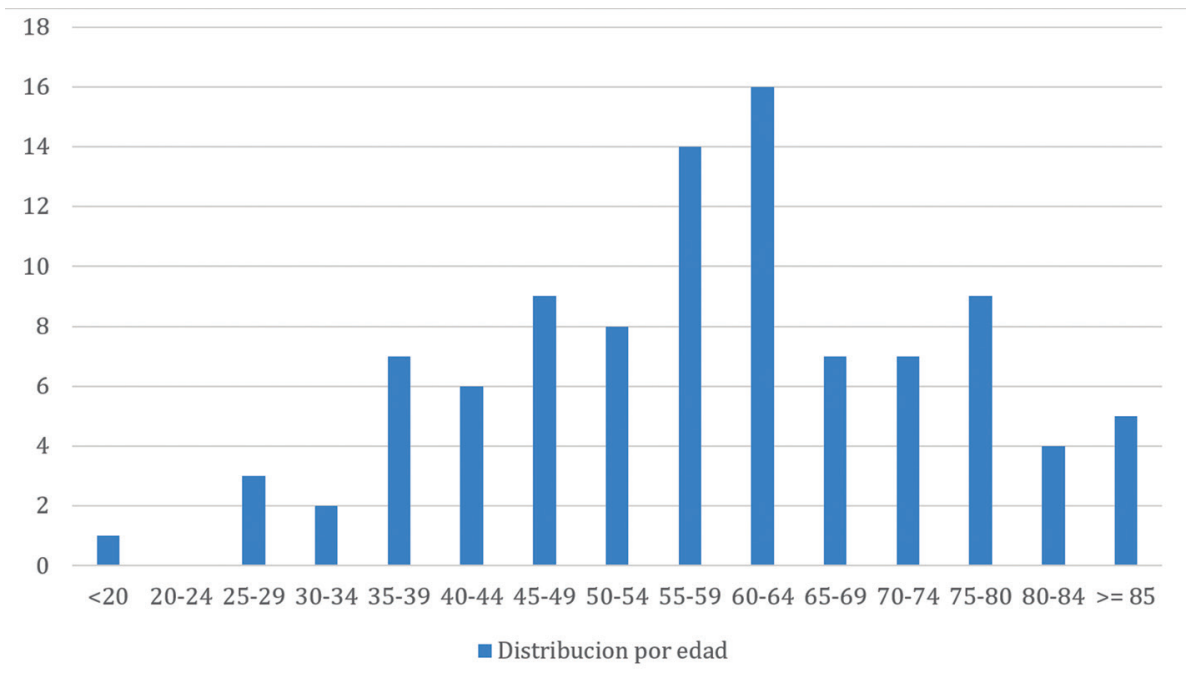

Figura 1. Distribución por edad de los casos de melanoma hallados en el estudio 


\begin{tabular}{|lcl}
\hline & ESTADIO DEL MELANOMA & \\
\hline & & $n$ \\
\hline IA & 18 & 18.3 \\
\hline IB & 6 & 6.1 \\
\hline IIA & 8 & 8.1 \\
\hline IIB & 10 & 10.2 \\
\hline IIC & 6 & 6.1 \\
\hline III & 3 & 3.0 \\
\hline IV & 14 & 14.2 \\
\hline NC & 23 & 23.4 \\
\hline Total & 10 & 10.2 \\
\hline
\end{tabular}

Tabla 1. Estadio del melanoma.

NC: No clasificable

Se incluyeron 492 historias clínicas de pacientes atendidos en la Clínica de Tumores del Piel y Mucosas de la Universidad de Caldas; se descartaron 394 historias por tener otros diagnósticos. Finalmente, se analizaron 98 historias clínicas de pacientes con diagnóstico de melanoma maligno, cuatro de los cuales presentaron otros tumores primarios, para un total de 102 tumores analizados.

El rango de edad de los pacientes osciló entre los 26 y los 97 años en las mujeres, con un promedio de 58,3 años. El rango en los hombres estuvo entre los 11 y los 88 años, con un promedio de 58,9 años. Su distribución se detalla en la figura 1. El 58,1 \% fueron mujeres y el $41,8 \%$ fueron hombres.

En 71 pacientes se pudo describir el tiempo de evolución, el cual varió entre 3 y 240 meses, con una media de 29,6. El 3,9 \% consultó en los tres primeros meses, el $44,7 \%$, entre los 4 y los 12 meses, y el 51,3\%, después del primer año.

Seis casos se presentaron asociados con nevo melanocítico congénito, de los cuales tres eran de tamaño pequeño y, dos, de tamaño mediano. En un caso no fue posible determinar el tamaño.

En 88 pacientes se pudo establecer el estadio, el cual se muestra en la tabla 1; en 10 casos, no se precisó por ausencia de datos en las historias clínicas.

En la tabla 2, se presenta la localización anatómica del melanoma encontrada en este estudio; la más frecuente fue cabeza y cuello (37,2\%), seguida de manos y pies (palmas, plantas y lecho subungular) (26,4\%). El tipo de melanoma maligno más frecuente fue el melanoma lentiginoso distal (acral) con 25 casos (24,5\%),

\begin{tabular}{|lll|}
\hline \multicolumn{2}{|c}{ LOCALIZACIÓN ANATÓMICA DEL MELANOMA } & \% \\
\hline \multicolumn{1}{|c|}{ Localización anatómica n } & 38 & 37.2 \\
\hline Cabeza y cuello & 38 & 26.4 \\
\hline Palamas, plantas o lecho subungular & 27 & 13.7 \\
\hline Tronco & 14 & 4.9 \\
\hline Brazos y antebrazos & 5 & 7.8 \\
\hline Muslos y piernas & 8 & 1.9 \\
\hline Ocular & 2 & 1.9 \\
\hline Orogenital & 2 & 5.8 \\
\hline Primario desconocido & 6 & \\
\hline
\end{tabular}

Tabla 2. Localización anatómica del melanoma 
seguido del melanoma extensivo superficial con 24 casos (23,5\%), el nodular con 21 casos (20,5\%), el metastásico con tumor primario desconocido en 9 casos $(8,8 \%)$, el lentigo maligno con 8 casos $(7,8 \%)$, el de mucosas con 8 casos (7,8 \%), y el melanoma lentigo maligno con 5 casos (4,9\%); no hubo datos suficientes que permitieran determinar el tipo de melanoma en 2 casos (1,9\%).

Se encontró ulceración en 35 casos, aunque en 10 no fue pertinente, dado que correspondían a melanoma in situ o metastásicos con tumor primario desconocido, y en 7 casos no se pudo determinar por datos insuficientes.

En la tabla 3 se describe el nivel de invasión representado por el índice Breslow y el nivel de Clark; en 30,3\% de los casos, fue de o a $1 \mathrm{~mm}$, y el 37,2 \% tenían un nivel IV de Clark IV.

Se practicó tratamiento quirúrgico con intención curativa en 63 pacientes, cirugía paliativa en 10 pacientes, tratamiento paliativo con vaciamiento ganglionar en 12 casos, radioterapia en 8 , criocirugía en 5 y quimioterapia en 13; se postergó el tratamiento según los resultados de los estudios de extensión en 2 pacientes y no se supo el tratamiento en 3 .

Se presentaron metástasis en 35 pacientes, el $40 \%$ fueron viscerales, el 37,1 \%, ganglionares, y el 22,8 \%, viscerales y ganglionares. En la tabla 4 se presenta la localización de las metástasis.

\section{DISCUSIÓN}

En el presente trabajo, se encontró una mayor prevalencia de melanoma maligno en mujeres (58,1\%), hecho que no se modificó en el tiempo en los registros del Comité de Tumores de Manizales ${ }^{(6)}$, que concuerdan con lo hallado en otros estudios llevados a cabo en la población colombiana ${ }^{(3)}$ y con los reportes de Perú ${ }^{(12)}$, Brasil ${ }^{(13)}$ y Europa ${ }^{(14)}$, y que contrastan con la literatura científica estadounidense ${ }^{\left({ }^{15}\right)}$ y con el Registro Poblacional de Cali (1962-2007) ${ }^{(16)}$ donde la prevalencia de melanoma maligno fue mayor en hombres. Se ha documentado una mayor prevalencia e incidencia en personas mayores ${ }^{(16,17)}$, asociadas con una mayor expectativa de vida y de exposición a la luz solar; sin embargo, la edad de presentación es amplia sin un grupo exclusivo, tal como se reporta en este trabajo, con una variación entre los 11 y los 97 años, siendo mayor la incidencia en la sexta década de la vida en ambos sexos; hallazgos similares se han reportado en otros estudios adelantados en Colombia, Perú y Argentina ${ }^{(16-18)}$. El sitio más frecuente de localización fue la cabeza y

\begin{tabular}{|c|c|c|}
\hline \multicolumn{3}{|c|}{$\begin{array}{c}\text { ÍNDICE DE BRESLOW Y NIVELES DE CLARK } \\
\text { Índice de Breslow (mm) }\end{array}$} \\
\hline & & $\%$ \\
\hline $0-1,0$ & 31 & 30.3 \\
\hline $1,1-2$ & 16 & 15.6 \\
\hline $2,1-3$ & 14 & 13.7 \\
\hline $3,1-4$ & 10 & 12.0 \\
\hline$>4$ & 12 & 9.8 \\
\hline Sin datos & 19 & 18.6 \\
\hline \multicolumn{3}{|c|}{ Niveles de Clark* } \\
\hline 1 & 21 & 20.5 \\
\hline II & 6 & 5.8 \\
\hline III & 11 & 10.7 \\
\hline IV & 38 & 37.2 \\
\hline V & 7 & 6.8 \\
\hline Sin datos & 5 & 4.9 \\
\hline
\end{tabular}

Tabla 3. Índice de Breslow y niveles de Clark

* E $14(13,7 \%)$ casos no se determinaron, por corresponder a melanoma de mucosas o metastásico. 


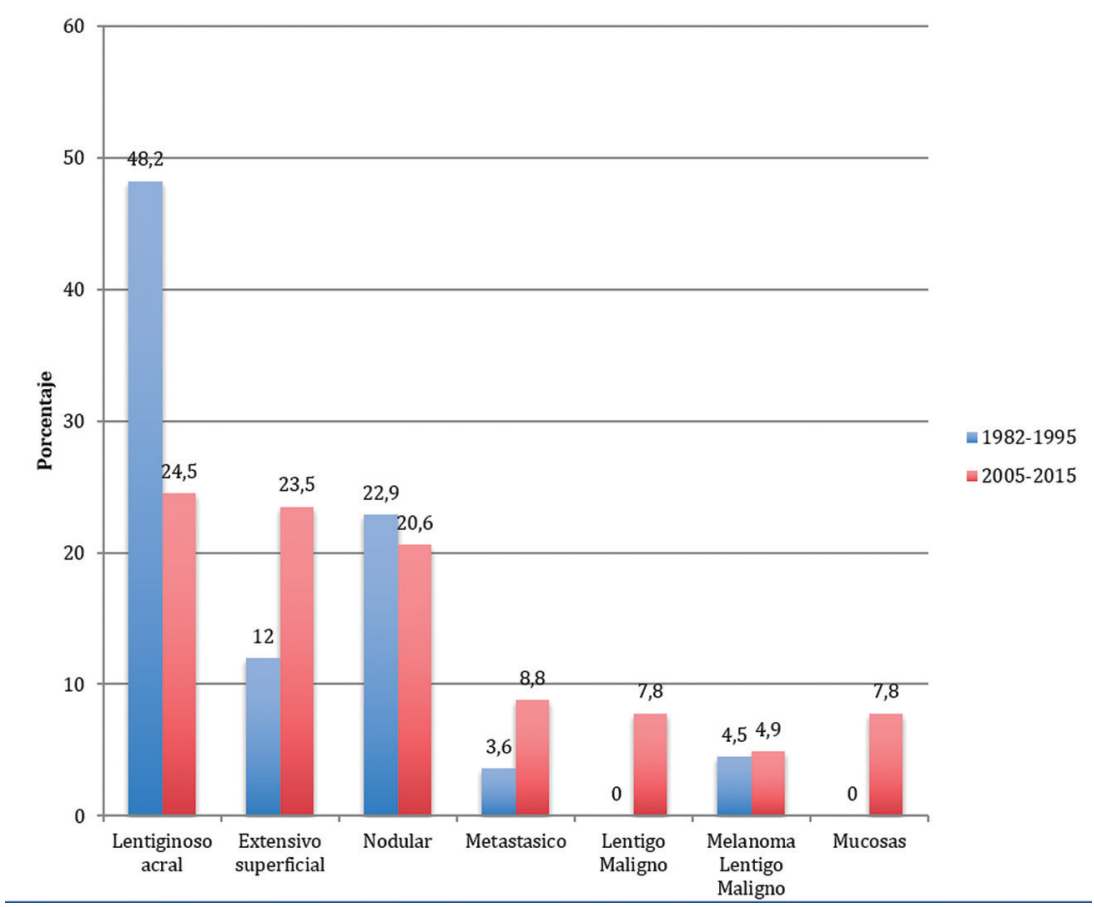

Figura 2. Comparación porcentual de los tipos de melanoma entre los dos periodos de los estudios comparados

\begin{tabular}{|lll|}
\hline LOCALIZACIÓN ANATÓMICA DE LAS METÁSTASIS \\
\hline \multicolumn{1}{|c|}{ Localización } & \multicolumn{1}{c|}{ (\%) } \\
\hline Piel & 12 & 34.2 \\
\hline Cerebro & 2 & 5.7 \\
\hline Pulmones & 6 & 17.1 \\
\hline Huesos & 2 & 5.7 \\
\hline Ovarios & 1 & 2.8 \\
\hline Epliplón & 1 & 2.8 \\
\hline Asas intestinales & 1 & 2.8 \\
\hline Labios mayores & 1 & 2.8 \\
\hline Órbitas & 1 & 2.8 \\
\hline
\end{tabular}

Tabla 4. Localización anatómica de las metástasis

el cuello, seguido de las palmas, las plantas y lechos subungulares; a diferencia de lo encontrado en los trabajos de Cali ${ }^{\left({ }^{(6)}\right.}$ y de otros países, como Perú ${ }^{(12)}$ Brasil $^{(13)}$ y Argentina ${ }^{(18)}$, con predominio de las lesiones ubicadas en el tronco y en las extremidades.

El tipo clínico e histológico más común fue el lentiginoso distal (acral), seguido por el extensivo superficial, tendencia que se ha mantenido en Caldas al compararlo con el estudio anterior (figura 2). Hallazgos similares se reportan entre orientales, negros, indios americanos y latinos residentes en Estados Unidos ${ }^{(6)}$, así como en otros estudios de Colombia ${ }^{(19)}$.

En este estudio, se encontró que el 5,8 \% de los casos están asociados con nevos melanocíticos congénitos, de tamaño pequeño y mediano, en $50 \%$ y 33,3 \% de los casos, respectivamente. Los pacientes con nevo melanocítico congénito pueden tener transformación maligna en un porcentaje substancialmente mayor que los 


\section{"En este estudio, se encontró que el 5,8\% de los casos están asociados con nevos melano- cíticos congénitos, de tamaño pequeño y mediano, en $50 \%$ y $33,3 \%$ de los casos, respectiva- mente."}

adquiridos ${ }^{(8)} \mathrm{y}$ el riesgo se presenta principalmente en los de tamaño gigante ${ }^{(9,20)}$. Sin embargo, a pesar de que los informes son controversiales y la relación causal ha sido difícil de establecer, también se reconoce el riesgo de melanoma en pacientes con nevos melanocíticos de tamaño pequeño y mediano, el cual va desde el o,8 hasta el 4,9\% ${ }^{(8,21)}$.

En el presente trabajo, la mayoría de los pacientes se encontraba en estadio IV, lo que podría asociarse con el gran número de casos de melanoma maligno nodular y metastásico; otro factor determinante sería la consulta tardía, ya que el $51 \%$ de los pacientes consultó después del primer año y, el $44 \%$, luego de 4 meses, similar a lo encontrado en el trabajo anterior. Se describen, como los principales motivos de consulta tardía por cáncer de piel en Caldas: el sexo femenino, la baja escolaridad, el régimen subsidiado de salud, la falta de certeza diagnóstica del médico general y el acceso tardío a la consulta especializada ${ }^{(22)}$.

En el 9,8 \% de los casos, no se logró establecer el estadio debido al subregistro de datos clínicos e histopatológicos, principalmente del índice de Breslow y la presencia o ausencia de ulceración; se destaca la importancia de la sistematización de estos datos para la realización de estudios posteriores.

En cuanto a las limitaciones del estudio, un hecho que se debe destacar es su carácter retrospectivo, con subregistro de variables importantes para determinar la clasificación y la supervivencia de los pacientes.

En conclusión, se encontró que, en la Clínica de Tumores, el melanoma maligno es más frecuente en las mujeres, con predominio del lentiginoso distal (acral), seguido del extensivo superficial y el nodular. En la mayoría de los casos, el diagnóstico fue tardío y el estadio IV fue el más frecuente.

Se sugiere la realización de estudios similares con una muestra poblacional que incluya diferentes departamentos y que permita establecer un perfil epidemiológico representativo del país.

\section{REFERENCIAS}

1. Frías AG, Ortiz HC, Lara HME. Estudio epidemiológico de melanoma maligno en el American British Cowdray Medical Center. An Med Asoc Med Hosp ABC. 2011;56:196-204.

2. Thompson JF, Scolyer RA, Kefford RF. Cutaneous (melanoma. Lancet. 2005;365:687-701.

3. Instituto Nacional de Cancerología. Estadisticas. Bogotá:Instituto Nacional de Cancerología; 2016. Fecha de consulta: 4 de abril de 2016. Disponible en: http://www.cancer.gov.co/cancer_en_cifras.

4. Pardo C, Cendales R. Incidencia, mortalidad y prevalencia de cáncer en Colombia, 2007-2011. Primera edición. Bogotá: Instituto Nacional de Cancerología; 2015. p.148.

5. Nova J, Sánchez G, Porras L. Cáncer de piel: perfil epidemiológico de un centro de referencia en Colombia, 2003-2005. Rev Salud Pública. 2007;9:595601.

6. Villegas MP, Jaramillo F. Comportamiento clínico, epidemiológico e histológico del melanoma maligno en el departamento de Caldas (Colombia). Rev Soc Colomb Dermatol. 1999;7:192-6.

7. Lavanderos J, Pérez JA, Jeria S, Concha D. Actualizaciones en melanoma maligno cutáneo. Cuadernos de Cirugía. 2010;24:47-56.

8. Acosta AE, Fierro E, Velásquez VE, Rueda X. Melanoma: patogénesis, clínica e histopatología. Rev Asoc Colomb Dermatol. 2009;17:87-108.

9. Bailey E, Sober A, Tsao H, Mihm M, Johnson T. Cutaneous melanoma. En: Fitzpatrick's Dermatology in General Medicine. New York: McGraw-Hill; 2012. p. 1416- 44 .

10. Joan AY, Díaz A, Montero JF, Jiménez L. Algunos factores pronósticos de interés en el melanoma maligno cutáneo. Rev Cubana Cir. 2010;49. Fecha de consulta: 21 de marzo de 2016. Disponible en: http://scieloprueba.sld.cu/scielo.php?script=sci_ arttext\&pid=So034-74932010000100001\&lng=es.

11. Balch CM, Gershenwald JE, Soong S, Thompsom JF, Atkins MB, Byrd DR. et al. Final version of 2009 AJCC melanoma staging and classification. J Clin Oncol. 2009;27:6199-206.

12. Gutiérrez C, Alarcón E, Valle R, Calderón G. Epidemiología del melanoma maligno en el Instituto Nacional de Enfermedades Neoplásicas, Perú, 2000-2004. Folia Dermatológica. 2007;18:23-7.

13. De Lima AS, Stein CE, Casemiro KP, Rovere RK. Epidemiology of melanoma in the South of Brazil: Study of a city in the Vale do Itajaí from 1999 to 2013. An Bras Dermatol. 2015;90:185-9. 
14. de Vries E, Coebergh JW. Cutaneous malignant melanoma in Europe. Eur J Cancer.2004;40:2355-66.

15. Rigel DS. Epidemiology of melanoma. Semin Cutan Med Surg. 2010;29:204-9.

16. Oliveros C, Bravo JC, Zambrano A, Cepeda M, Ramírez AF. Tendencias en la incidencia y la mortalidad en melanoma maligno en los últimos 60 años y análisis de los datos del registro poblacional de cáncer de Cali. Rev Asoc Colomb Dermatol. 2012;20:255-64.

17. Guy GP, Jr, Thomas CC, Thompson T, Watson M, Massetti GM, Richardson LC. Vital signs: Melanoma incidence and mortality trends and projections-United States, 1982-2030. MMWR Morb Mortal Wkly Rep. 2015;64:591-6.

18. Loria DI, González A, Latorre C. Epidemiología del melanoma cutáneo en Argentina: análisis del Registro Argentino de Melanoma Cutáneo. Dermatología Argentina. 2010;16:39-45.

19. Pozzobon F, Acosta A, Carreño A, Fierro E. Características del melanoma cutáneo primario en el Instituto Nacional de Cancerología, 2006-2010. Rev Colomb Cancerol. 2013;17:111-8.

20. Rolón M, Arredondo M. Melanoma originado en nevus melanocítico congénito. Rev Asoc Colomb Dermatol. 2008;16:305-6.

21. Newton JA. Melanocytic Naevi and Melanoma. En: Harper J, Oranje A, Prose N. Textbook of pediatric dermatology. Third edition. Oxford: Blackwell Publishing; 2011: 1230-57.

22. Mesa J, Jaramillo F, Benjumea M. Factores relacionados con la consulta tardía a dermatología por cáncer de piel. Prueba piloto. Hacia la Promoción en Salud. 2008;13:178-94. 